\title{
Experimental Drift Mapping of Indian Ocean Gyre Aircraft Debris
}

\author{
Lev M. Vozchikov, Lab Selena \\ Student Transportation of America, USA \\ Email: Ivselena@aol.com
}

Received 29 December 2015; accepted 23 February 2016; published 26 February 2016

Copyright (C) 2016 by authors and Scientific Research Publishing Inc.

This work is licensed under the Creative Commons Attribution International License (CC BY). http://creativecommons.org/licenses/by/4.0/

c) (i) Open Access

\section{Abstract}

A graphical simulation is developed to calculate the map of the Indian Ocean debris drift, and using the experimental approach to study the alternative crash location of the vanished aircraft MH370. Drawing research used new discovered and known statistics of Oceanic surface currents map, and published satellite data of aircraft tracking to review the new critical data. Theory of prediction in a research for lost transport unit is the successful tool to control ongoing investigation. The entire area of the crash location of plane's debris drift model is based on the early stage of the Oceanic mapping of underwater investigation. With discovering actual new facts, ongoing efforts are in stage to evaluate interpret factual findings. Appearance of MH370 aircraft wing part-flaperon ignited research trends of Indian Ocean debris drift mapping study with the purpose to isolate final crash location. On experimental basis, the article discusses the comparison of ongoing research of international groups in aim to point underwater search to the the site of plane crash. The process of Oceanic debris transport is a physics task, and the computation of debris drift can be observed on the interval statistics data set of Oceanic debris season. In this experimental simulation, on the base of Indian Ocean drift data-observer studied the possibility of the North Gyre debris transport trace to explain the timeout of single appearance of flaperon, declared in official investigation. The article resumes to study and to conclude the possible alternative approach of North Gyre crash location. Graphical computer visualization presented in a comparison to recent studies is conducted by Australian and Germany groups. Their trends on the scour survey of the first phase stage seabed mapping, oceanic surface search in a mean time, can be described as comparable approximations. Given in a research, experimental simulation graphically visualized the possibility of North Gyre debris transport exchange.

\section{Keywords}

Debris, Drift Simulation, Maritime, Transport 


\section{Introduction}

Oceanic map drifting debris, developed by Australian MH370 marina seabed investigation team, had predicted with a delay arriving of the wreckage debris at French Reunion Island as a single rare event. Waiting plane's debris appearance of New Zealand coast is concluded as a developmental approach. Followed the founded plane wing flaperon, GEOMAR, Helmholtz Centre for Oceanic Research Kiel, Germany studied and summarized debris mapping in an equator area of South Indian Ocean. Their development affected the possible search area definition; as the research studied non-cosmopolitan barnacles, the flaperon residue [2]. Factual delay in an estimate floating debris trace exists in the interval 3 - 9 months [1]. Appearance timing of the single debris is an explainable in a junction with a determination of the physics properties of the flaperon wreckage, recent discovered off the coast of Madagascar. In the approach of the casual known entire trace start point-the satellite South Arc $7^{\text {th }}$, we could conclude that the flaperon was floating with greater speed in waters than expected, compared different simulations mapping of Arc $7^{\text {th }}$ to explain the debris drift delay of water transport. North Indian Ocean Gyre/Bengal Gyre has currents with higher velocity, at least, several times greater than South Indian Ocean Gyre [3] [4]. Effective calculated simulation should find out the possible start point within Indian Ocean to model the map of floating debris. Statistically, successful modeling can be even within short season-periodic interval. Partially, North Indian Gyre stream is the actual currents additives source which is to speed up floating ruble trace in an Oceanic Water Stream [4]. The experimental simulation of debris map shows the assumed North Indian Gyre start point as possible interpretation debris drift timing. The initial area search, based on flight path missing $\mathrm{MH}$ aircraft, includes satellite tracking data $7^{\text {th }}$ Arc crossed Bengal Bay at North and approaching coast of Australia [5].

\section{Experimental Simulation}

The season map of currents, and surface winds of the Oceanic region - the data observer, are the countable arguments in a graphical drawing, tracing winds development, occurred casualties in a debris drift map simulation modeling. Different start points comparison in an experimental Oceanic drift simulation study of the North Gyre and the South Gyre prototypes allow approach to actual computation, and provide improving explanation of outline of the initial drift debris mapping. Oceanic South Indian area is taken to count waiting time of debris transported across Indian Ocean. Scheduled debris arriving to Madagascar off coast located at Reunion Island in an Australian drift map version is in time-out in a comparison with the basic model parameters. Several months' observed delay needs to explain.

Alternative start point debris mapping explanation was assumed to verify the North Gyre area, the first stage area search, as a possible flaperon crash location.

Assumption points to the research of the first phase aircraft investigation in a Bengal Bay area. Aircraft related underwater anomaly appearance in this region was dated in March 2014, reported by Geo Survey images, studied in an emerging technology laboratory. Image color reconstruction returns the identification anomaly as the wreckage of the searching aircraft [7]. The argument taken in account for an actual verification map of the Oceanic transport of crash debris is found.

Definition "drifter" given to the moving rubble means that it is a freely float, drifting with the oceanic water currents stream. Actual flaperon laboratory study result shows that it has unique construction that made it floating in an Oceanic saltwater. The flaperon is the good floating aircraft wing chunk. In accordance with Newton, Archimedes laws that the water accelerating equation parameters are the salt-water density, currents speeds, and flaperon material density. There was calculated the wing flap construction ability to float. Unique single part has an ability to float in according to the Newton Law, and capacity of the floating ability in a salt-water makes this part an ideal drifter. Such ability is the physic property of this wing part, and it is original quality of the construction material which this part made. Total flaperon weight, its construction elements mass, equals to the mass of the salt water which flaperon pushes out in position of float.

The physics debris parameters may has cause different floating ability, and the sizes, objects properties that are not predictable before wreckage are found. Nevertheless, partial result of such assumption found argumentation in different tracking lengths, and different speeds proposed floating debris traces, basic formal model.

Actual difference in a predicted time of the arriving to Reunion Island of the flaperon [6] gave us a reason to suggest that it was floating with higher velocity currents of the North Gyre [4], substitute trace. Based on such assumption, there was set two correlated start points that produced positive comparative results. We can see on Figure 1 and Figure 2 that the both emulations produce the Madagascar drifter traces. If the Australia of coast simulation studied [1]-[3], Bengal Gyre is presented the significant new result. Results of experimental simulation visual comparison set us in due to assume that the North Indian Ocean trace is possible to follow the 


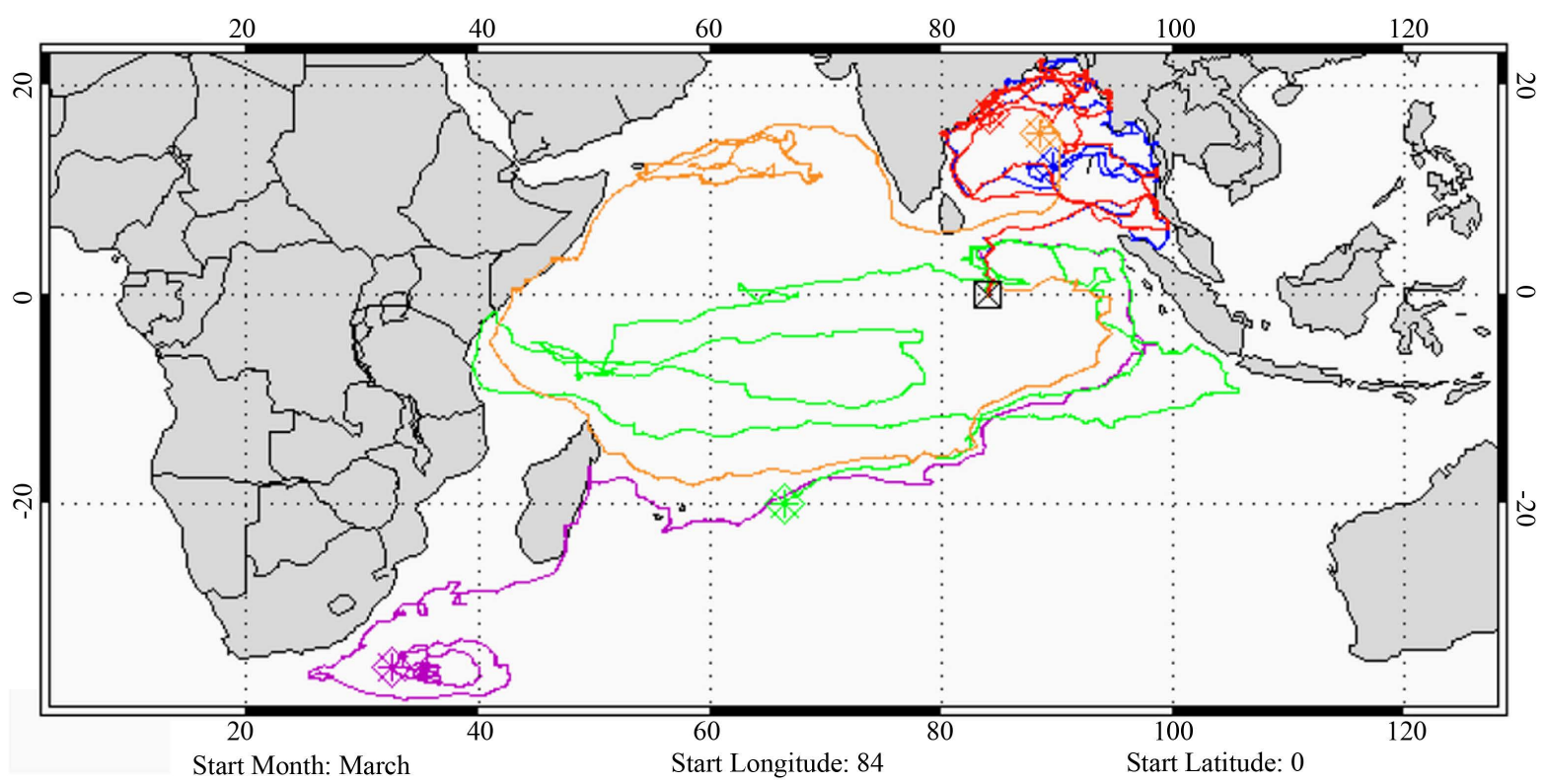

Figure 1. North Indian Ocean. Bengal Gyre.

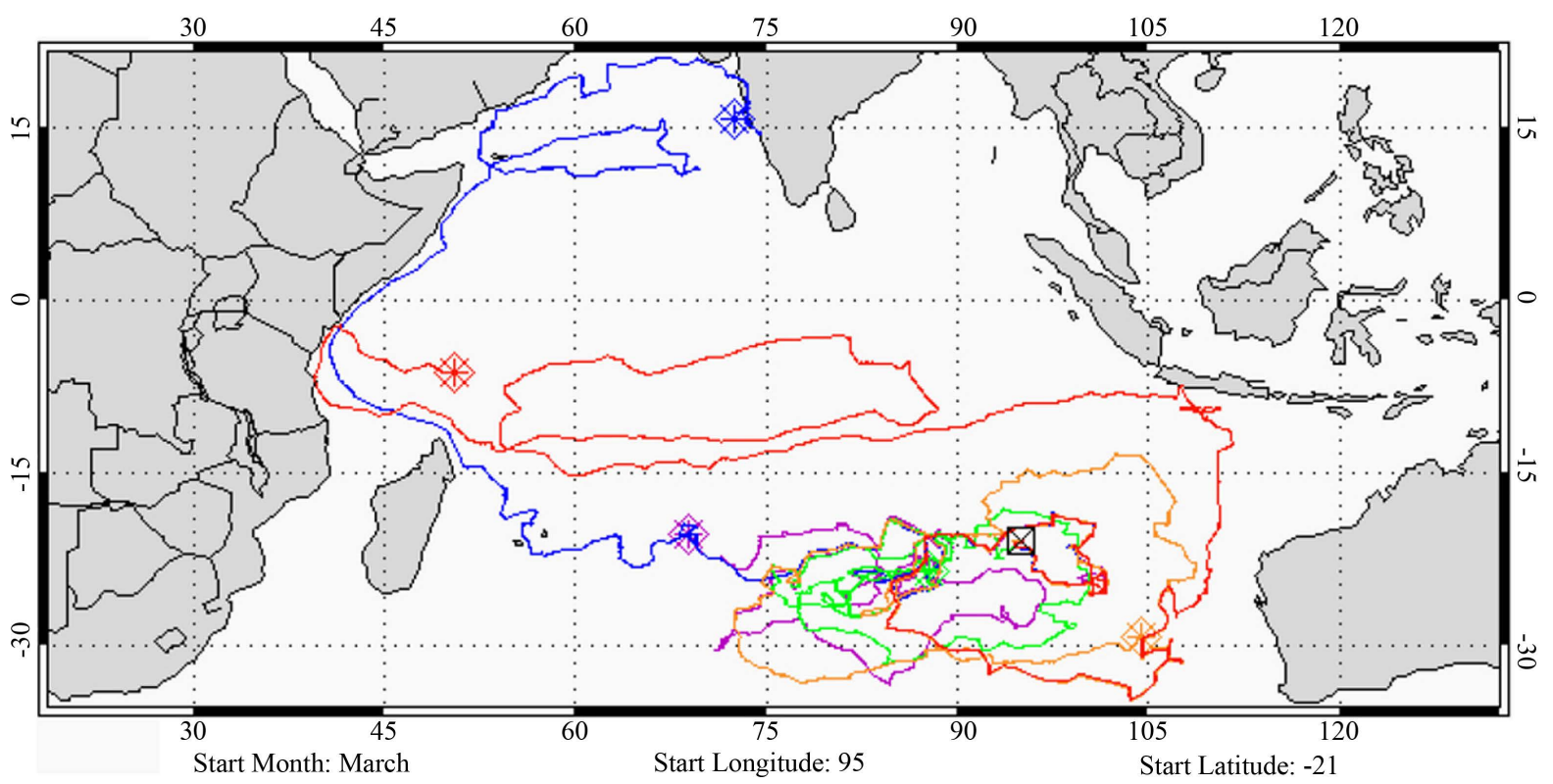

Figure 2. South Indian Ocean Gyre.

objective to optimize the waiting of the debris map schedule.

\section{Result}

Calculation developed on the drift ship simulator NASA Oceanic model ship drift “Ocean Motion” made in a year season range 2014-2015 years, successfully showed several alternative possible tracing drifts results on the developed drawings Figure 1-NIO/North Indian Ocean/, Figure 2-SIO/South Indian Ocean/. Figure 1 and Figure 2 show Indian Ocean drift graphical simulation mapping result. Here shown in a comparison both start SIO and NIO points simulations, the North Ocean Gyre and the South Ocean Gyre, includes mapping results, Madagascar of coast drifters traces/pink mark. Simulator allows visually in a color drawing to present 5 random drifters of season statistics currents trends. With simulator help, there was possible to determine 2 start points 
simulation off the Australian Coast, and Bengal Bay traced to the point of the found debris at the Reunion Island. These alternatives in a general assumption are different from each other by the drift schedule.

Both points are located on the $7^{\text {th }}$ Arc, the Satellite INMARSAT trace of the vanished aircraft. In a comparison, we can show that there is a strong possibility of the North Arc trace start point prediction because the observer data point to the stream of the currents in a North Indian Ocean (see Figure 1, North Indian Ocean Gyre). Simulation is show the higher speed transport agent in a comparison with South Indian Ocean (see Figure 2, South Indian Ocean Gyre). The South Indian Ocean start point simulation, off the Australia coast, is the formal base stimulation made of ongoing phase investigation [1].

Ship drift data observer simulator has tasked to emulate drawings in two different cases with start points in North and South segments of Arc 7th. Experimental simulation results presented the drifters traces tracking toward the coast of Madagascar. Significant result is the presence of equal drift traces toward Reunion Island. Complex character Oceanic Currents is responsible for additives stream debris transport. Each drift simulation consists of 5 drifters-observers, selective colored in a drawings, we can see the Reunion Island trace, marked in pink is exist within both drift data observers showed in Figure 1 and Figure 2. Alternative experimental mapping has show the possible substitution North 7th segment as a wreckage search area.

\section{Conclusion}

Graphical visualization on Figure 1 and Figure 2 illustrates the result of experimental simulation; and depicts North and South start tracing points as equal traces which have debris transport appearance to the Madagascar coast. Assumed possible drift trace within an improved model quality found in an experiment of simulation drift start point-Bengal Gyre of North Indian Ocean, within $7^{\text {th }}$ Arc location, declared the search area at the first phase plane search. Debris drift delay, observed in the first mapping attempt, taken as an argument for mapping aircraft debris drift models in a comparison-is necessary for determination of actual delay, the length of the rubble drift, to compare with the current debris drift properties found. Verification simulation result with real-time measurement is the factual basis which allows us to alternate the modification of start point in an experiment. Based on the study results, researcher should assume complex additive trace for alternate location of the geographical start point of the simulation debris transport - in an aim to trace substitution currents in a North part of Indian Ocean. In a research, we have accounted several argumentations which point us to the possible North Indian Ocean trace debris drift. The measurement of debris traces lengths, based on the North Indian Ocean partway prototype, definitely points to interpretation of the North 7th Arc as the possible start point alternate simulation. This region is geographically consistent with an entire approach to submerge area search wreckage. North 7th Arc area was in search state from March 8, 2014 year when the MH370 aircraft disappeared. Oceanic surface debris search is conducted due to active black boxes period of the lost MH370 aircraft.

\section{Summary}

Experiment-based successful outcome established an alternative map of the drift simulation which pointed the crash location within 7th Arc of the Oceanic area. Meant data visualization modeling provided by NASA "Ocean Motion" simulator resulted in the several random statistical ship drift traces.

Rare appearance of the single wing part debris lost aircraft wreckage within predicted debris mapping delayed and found explanation in the North Indian Oceanic transport currents complex. First here was assumption that the alternative trace start point was possible to locate on the $7^{\text {th }}$ Arc area, and then simulation returned positive result for the North Indian Ocean.

The presented research shows the several significant factual findings which have to argue that the Bengal Gyre is a possible point of the final flaperon crash location. Actual absence aircraft debris, long measure flaperon debris appearance, positive resulted alternative research crash location discussed in article.

Essential recognizable image color reconstruction of the Geo Resonance Ltd., pictures of Bengal Bay GeoSurvey identified specific wreckage aircraft properties. Color research conducted in an innovation trend to study presented author emerging color technology developed to facilitate industrial visualization image [7].

North Indian Ocean area declared aircraft lost zone in a beginning phase search of disappeared MH370 plane. Initiated investigation returned no result in an airplane Black Boxes search in a 90 days period then resumed equipment-Black boxes fault by the battery expiration date. Then switched investigation phase-out continued in the South area of 7th Arc. Nevertheless, the study of new reliable facts provides determination to group the 
North and the South search paths as concerns of the JACC, ATSB investigation efforts.

\section{References}

[1] MH370 Aircraft Debris and Debris Modeling, 2015. Educational Fact Sheet, ATSB AU CSIRO, Aviation Publication, 4.

[2] Durgadoo, J. and Biastoch, A. (2015) Where Is MH370? GEOMAR Helmholtz Centre for Oceanic Research Kiel, Press Release 44, 2015. http://www.geomar.de/en/service/kommunikation/singlepm/article/wo-ist-mh370

[3] Maximenko, N. and Hafner, J. (2015) Marine Debris Drift Model Simulates MH370 Crash Site and Flow Paths. IPRC, Honolulu.

[4] Lumpkin, R. and Johnson, G.C. (2013) Global Ocean Surface Velocities from Drifters: Mean, Variance, ENSO Response, and Seasonal Cycle. Journal of Geophysical Research: Oceans, 118, 2992-3006.

[5] Ashton, C., Bruce, A.S., Colledge, G. and Dickinson, M. (2015) The Search for MH370. The Journal of Navigation, 68, 1-22. http://dx.doi.org/10.1017/S037346331400068X

[6] MH370-Definition of Underwater Search Areas, 2014, ATSB Transport Safety Report, External Aviation Investigation AE-2014-054.

[7] Vozchikov, L. (2015) Color Design Research. Color Cell. Third 21st Century Academic Forum, Conference at Harvard, Harvard University, Boston, 21-22 September 2015, 15. http://www.21caf.org/3rd-hrd-conference-program.html 